\section{ANIMAT: A set of programs to generate, edit, and display sequences of vector-based images}

\section{ALLEN A. MONTGOMERY and GILBERT SOO HOO Army Audiology and Speech Center \\ Walter Reed Army Medical Center Washington, D.C. 20012}

A set of programs has been developed to enable a user to perform a variety of graphic operations. These operations, although oriented toward animated display, constitute a general set of procedures for displaying stimuli useful in a variety of experiments in visual perception, memory, and masking. The capabilities include generating and editing images, creating sequences of slowly changing images for animation, and displaying rapid sequences of images with a variety of options for timing, sequencing, overlapping, and temporal patterning.

Environment. The programs are in FORTRAN and run on an intelligent stand-alone graphics system with flexible disks and $64 \mathrm{~K}$ bytes of core (Tektronix, Model 4081). The display is in storage, refresh, or mixed mode under program control. The routines operate primarily on images stored as assemblies of vectors in a form that permits rapid refreshed display known as a plot (PLT) file. PLT files that have only absolute vectors and contain the same number of vectors are said to be conformable and are used in the generation of animated sequences. In general, each PLT file contains one image and is loaded prior to use from mass storage to the display memory for immediate access.

The display medium is a 19-in. (diagonal) CRT with medium-to-rapid decay phosphor (similar to P31 when operating in refresh mode). In a typical animation sequence, 35 130-vector images are presented at 40 frames/sec under preprogrammed or interactive keyboard control.

ANIMAT. The programs in ANIMAT are broken down into five groups according to function.

1. Programs to create new PLT files. MAKPLT generates a PLT file with joystick and displays an image as created. A new file can be appended to the existing file. SUBR PLTGEN accepts $x, y$ and op-code arrays from the main program, prompts the file name, and generates the PLT file up to 200 vectors (the limit is easily increased).

2. Programs to read, display, and edit existing PLT files. PLTPRE is the main program to read and display vector coordinates of a PLT file. PLTMOD generates a

Software development received financial support from the Department of Clinical Investigation, Walter Reed Army Medical Center, under Work Unit 2525. The opinions or assertions contained herein are the private views of the authors and are not to be construed as official or as reflecting the views of the Department of the Army or the Department of Defense. new file or modifies an existing file. The user can add, delete, or change vector values and optionally rename a file. All relative vectors are changed to absolute vectors for subsequent ease of computation, and optional translation of axes may be performed. PLTRED reads the existing PLT file in 128-size buffers and displays each buffer as an image and as an integer array. PLTRED allows careful examination of the image and the location of erroneous vectors. PLTDMP reads and displays numeric contents (not graphics) of an existing PLT file. This function permits detailed examination of real and absolute vectors and detection of other vector codes. PLTDMP also provides the vector count and location of invisible moves.

3. Programs to interpolate smoothly between two existing files. ALSPLT reads two existing, conformable files, generates $\mathrm{N}$ frames equally spaced, and linearly interpolates between the shapes contained on the existing files. Images up to 200 vectors may be generated. PLTGEN is called to create files after vectors are calculated. MICAM accepts up to five existing files, modifies shapes according to geometric influence of surrounding shapes if desired, and interpolates between those new images to create animated transitions through the five targets. This program is used to create longer animated sequences.

4. Programs to display sequences of frames (animation). LMUP7, the main program, calls PLTOBJ (an assembly language subroutine supplied by Tektronix) to read files and pack them into display memory. LMUP7 reads the name file of names, loads files $(\leqslant 35$ different images), displays them with various user options for number of repetitions and timing, and allows the user to resequence display up to 180 frame displays (approximately $4 \mathrm{sec}$ ).

5. Miscellaneous programs. VLR accepts two conformable files of closed shapes centered about a common origin, calculates the areas, and compares the shapes in two ways: (a) by computing the mean slope difference of corresponding vectors between the two images and (b) by calculating the nonoverlapping area. JOY 3 moves a file around under joystick control and then, optionally, creates a new file with the transformed coordinates. ATBBTA converts the ASCII PLT file to binary or binary to ASCII for transfer to another computer. TEST I permits the user to measure exact display times for specific files and calculate temporal characteristics of display sequences.

Input/Output. Graphic input is accomplished in one of four ways. (1) An image may be traced, vector by vector, on a graphic tablet to convert the file to PLT format. (2) By generating $x$ and $y$ arrays internally with a user-supplied FORTRAN program, subroutine PLTGEN can be used to create the corresponding PLT file for later display or editing. For example, if the equa- 
tion $y=f(x)$ for an ellipse was evaluated at 20 values of $x$, a 20 -vector ellipse could be generated. (3) Running MAKPLT and using the joystick creates an image by hand that is then stored as a PLT file. (4) ALSPLT or MICAM generates images that are interpolations between two existing images. For example, varying degrees of "roundness" could be created by interpolating between a circle and a square.

Graphic output is normally displayed on the CRT as a stored or refreshed image, but hard copies are available. The CRT may be videotaped for noninteractive display at remote locations if the temporal characteristics of the display are not obscured by the frame rate of the videotape recorder.

Availability. A listing of all sources and supporting documentation is available at no cost. Tektronix 4081 users may send a formatted 8-in. flexible disk (hard sector) or .25-in. cassette tape for a copy of sources and execu table object modules.

Portability. All routines will run without difficulty on a Tektronix Model 4081 graphics system with $64 \mathrm{~K}$ bytes of memory under GOS V.4. The algorithms for interpolation between images and sequencing of displays (ALSPLT and LMUP7) are the least machine dependent and should be adaptable to other systems with refresh capability. The routines to manipulate and store PLT files, unless modified, are not likely to be useful outside a Tektronix environment. In all sources the graphic I/O sections and other machine-dependent features are clearly labeled.

(Accepted for publication November 25, 1981.) 